\title{
Changing trends in antibiotic susceptibility pattern among clinical isolates of Pseudomonas species in a tertiary care hospital in Nepal
}

\author{
Hamal Deependra ${ }^{1}$, Bhatta Dharm Raj ${ }^{1}$, Shrestha Rajani ${ }^{1}$, HS Supram ${ }^{1}$, Nayak Niranjan ${ }^{1}$, \\ Gokhale Shishir ${ }^{1}$, Parajuli Sulochana ${ }^{1}$
}

\author{
Affiliations: \\ ${ }^{1}$ Department of Microbiology, Manipal College of \\ Medical Sciences, Pokhara, Nepal
}

\section{Correspondence to:}

Deependra Hamal

Department of Microbiology, Manipal College of Medical Sciences, Pokhara, Nepal

Email: hmldipendra@yahoo.com

Received: 24 July, 2021 Accepted: 5 Sept, 2021

How to cite this Article:

Hamal D, Bhatta DR, Shrestha R, HS Supram, Nayak $\mathrm{N}$, Gokhale S, Parajuli S. Changing trends in antibiotic susceptibility pattern among clinical isolates of Pseudomonas species in a tertiary care hospital in Nepal. Ann. Clin. Chem. Lab. Med. 2021:4(1); 6-12

DOI: https://doi.org/10.3126/acclm.v4i1.42674

(C) 2022 Nepalese Association for Clinical Chemistry

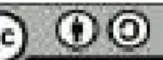

\footnotetext{
This work is licensed under a Creative Commons Attribution-Share Alike 4.0 Interna-
} tional License.

\begin{abstract}
BACKGROUND

Pseudomonas spp is notoriously known to be important agent of hospital as well as community acquired infections. These organisms are resistant to many antibiotics by intrinsic and acquired mechanisms making infections difficult to treat. Regular surveillance of infection and antibiotic resistance patterns is necessary for selection of appropriate antibiotics for treatment.

\section{METHODS}

Non repeating Pseudomonas spp isolated from all clinical samples during the year 2017-2019 were included in the study. Identification of Isolates were done by standard conventional tests and antibiotics sensitivity test was done by Kirby-Bauer disk diffusion method following the CLSI guidelines, 2017.
\end{abstract}

\section{RESULTS}

A total of 42,545 specimens comprising of sputum, blood, pus, urine, body fluids and endotracheal tubes were processed during the entire 3 year period. There was significant increase ( $\mathrm{p}$-value $<0.05$ ) in yearly isolation rate of Pseudomonas spp i.e. 141(7.95\%), 197 $(12.14 \%)$ and $303(15.69 \%)$ isolates in the year 2017 , 2018, and 2019 respectively. Resistance rates of the isolates to cefoparazone sulbactam, Piperacillin Tazobactam, and Tobramycin was significantly decreasing ( $p$-value $<0.05$ ). Overall resistance to ceftazidime $(66.13 \%)$ was alarming. Decrease in MDR isolates were statistically significant over 3 years e.g. 32 $(22.69 \%), 31(15.73 \%)$ and $37(12.21 \%)$ isolates in the year 2017, 2018 and 2019 respectively (p-value $<0.05$ ).

\section{CONCLUSION}

The rate of infection of Pseudomonas spp is significantly increasing. There was gradual decrease in number of resistant isolates and MDR isolates over the period starting from 2017 to 2019 which is a favorable trend. The possible factors playing role have to be further studied, identified and promoted.

KEY WORDS:

Antibiotic resistance, Pseudomonas spp 


\section{INTRODUCTION}

Pseudomonas species, member of the family Pseudomonadaceae, is an aerobic, gram negative bacilli. It is saprophyte, commonly found in soil, and water, but have emerged as important human pathogen. Pseudomonas spp is a notorious nosocomial pathogen ${ }^{1}$. It is gaining importance as a cause of community acquired pneumonia, blood stream infection, wound infections, including those of burn wounds. Pathogenicity of Pseudomonas is associated with numerous virulence factors like liposaccharide, type VI secretion system, biofilm production, exotoxin $\mathrm{A}$, proteases and alginate production, etc. ${ }^{2}$.

Antibiotic therapy of Pseudomonas infections is challenging as these are intrinsically resistant to a number of antibiotics, carry genes for antibiotic resistance and have adapted multiple mechanisms of antibiotic resistance ${ }^{3}$. Even, resistance among Pseudomonas spp to carbapenem, the last resort antibibiotic, is on steady increase. This trait of intrinsic and acquired antibiotic resistance has narrowed down the choice of antibiotics and clinician has to rely on more toxic and more expensive antibiotics. In context to Nepal, authors like Baral et al, Yadav et al have reported MDR Pseudomonas as high as $60.1 \%, 73.3 \%$ respectively and are associated with prolonged hospital stay and increased mortality which is a serious situation $^{4,5}$. Such emergence of multidrug resistance (MDR), and carbepenem resistance are of primary concern globally, as these factors are importantly related to prolonged hospital stay, increased morbidity and mortality ${ }^{6}$.

The rate of infection by Pseudomonas spp vary in different clinical settings. There is a need of proper surveillance on occurrence of infection by Pseudomonas and their pattern of antibiotic susceptibility. This will guide clinicians to choose the best antibiotics for empirical therapy without delay. This study was, therefore, aimed to find out the infection rate due to Pseudomonas spp and determine the changing trend in antibiotic resistance pattern among the isolates.

\section{METHOD}

\section{Setting and Design}

This is an observational and descriptive study conducted at the tertiary care hospital located in Nepal. The culture and sensitivity data of non-repeating isolates of Pseudomonas spp for 3 consecutive years (2017, 2018 and 2019) formed the material of the study.

\section{Isolation and identification of Organism}

All the clinical samples obtained in microbiological laboratory were processed by standard microbiological techniques. The specimens had been cultured on Chocolate agar, 5\% Sheep Blood agar, MacConkey agar plates and biphasic media (Hi-Media, India) based on type of clinical samples. Isolates were identified as Pseudomonas spp by standard, conventional phenotypic methods using Triple sugar iron agar(TSI), Sulphur Indole Motility agar(SIM), Christensen's urease agar, Simmons citrate agar, oxidase strip and pigment production ${ }^{7}$.

\section{Antibiotic sensitivity testing}

Antibiotic sensitivity tests against Gentamicin $(10 \mu \mathrm{g})$, Amikacin $(30 \mu \mathrm{g})$, Tobramycin $(10 \mu \mathrm{g})$, Ciprofloxacin $(5 \mu \mathrm{g})$, Ceftazidime $(30 \mu \mathrm{g})$, Piperacilin-Tazobactam $(100 / 10 \mu \mathrm{g})$, Cefoperazone$\operatorname{sulbactam}(75 / 10 \quad \mu \mathrm{g}), \quad$ Colistin(10 $\mu \mathrm{g})$, Polymixin B(300 units), and Imipenem(10 $\mu \mathrm{g})$ (Hi-Media, India) were performed by KirbyBauer disk diffusion method and results were recorded and Interpreted in accordance with the criteria laid down by Clinical Laboratory Standard Institute (CLSI), $2017^{8}$. As, antibiotics like colistin, cefoperazone-sulbactam and polymixin B don't have CLSI interpretative criteria for disk diffusion antibiotic susceptibility test, guidelines given by Hi-Media laboratories was used for interpretation ${ }^{9}$. Multidrug resistance was defined according to the current guidelines given jointly by the European Centre for Disease Prevention and Control (ECDC) and the Centers for Disease Control and Prevention $(\mathrm{CDC})^{10}$.

\section{Statistical Analysis}

Data were analyzed by SPSS version 20. Categorical variables were calculated as percentages. Chi-square test was used to compare two groups. All $\mathrm{p}$ values $<0.05$ were considered as statistically significant.

\section{RESULTS}

A total of 42,545 specimens comprising of sputum, blood, pus, urine, body fluids and endotracheal tubes were processed during the entire 
three year period. Of these, 13675 specimens were processed in the year 2017, 13780 specimens in 2018, and 15090 in 2019, which yielded 1772, 1622 and 1930 gram negative bacilli respectively. Out of these, Pseudomonas spp accounted for 141(7.95\%), isolates in the year 2017, 197(12.14\%) isolates in 2018, and 303(15.69\%) in 2019. The isolation rate of Pseudomonas spp was found to have significantly increased ( $p$-value $<0.05$ ) over the years.

Table 1: Gender, age and season wise distribution of Pseudomonas spp.

\begin{tabular}{l|l|l|l|l|}
\cline { 2 - 5 } & \multicolumn{5}{|c|}{ Year of isolation } \\
\hline Gender & 2017 & 2018 & 2019 & Total \\
\hline Male & $90(63.83 \%)$ & $125(63.45 \%)$ & $170(56.1 \%)$ & $385(60.06 \%)$ \\
\hline Female & $51(36.17 \%)$ & $72(36.55 \%)$ & $133(43.89 \%)$ & $256(39.93 \%)$ \\
\hline Total & 141 & 197 & 303 & 641 \\
\hline Age & & & & \\
\hline $0-14$ year & 24 & 44 & 75 & $143(22.3 \%)$ \\
\hline $15-60$ year & 74 & 91 & 139 & $304(47.42 \%)$ \\
\hline$>60$ year & 43 & 62 & 89 & $194(30.26 \%)$ \\
\hline Total & 141 & 197 & 303 & 641 \\
\hline Season & & & & $371(57.87 \%)$ \\
\hline Rainy season & 98 & 102 & 171 & $270(42.12 \%)$ \\
\hline Other season & 43 & 95 & 132 & 641 \\
\hline Total & 141 & 197 & 303 & \\
\hline
\end{tabular}

Distribution of Pseudomonas spp according to gender, age and season is shown in table no 1. It was found that, in all 3 years, numbers of males suffering from Pseudomonas infection were significantly higher compared to females. Pseudomonas spp was isolated from subjects belonging to all age groups starting from neonates to individuals as old as or even older than 80 years. It was noticed that majority of Pseudomonas i.e. 47.42\% (304/641) were isolated from adults (15-60 years of age), while 194(30.26\%) and 143(22.30\%) were from individuals of old age group ( $>60$ years) and those of younger age group (0-14 years) respectively.

Pseudomonas spp isolation rate was more during the rainy season i.e. 371(57.8\%) when compared with other months $270(42.12 \%)$ (p-value $>0.05)$.

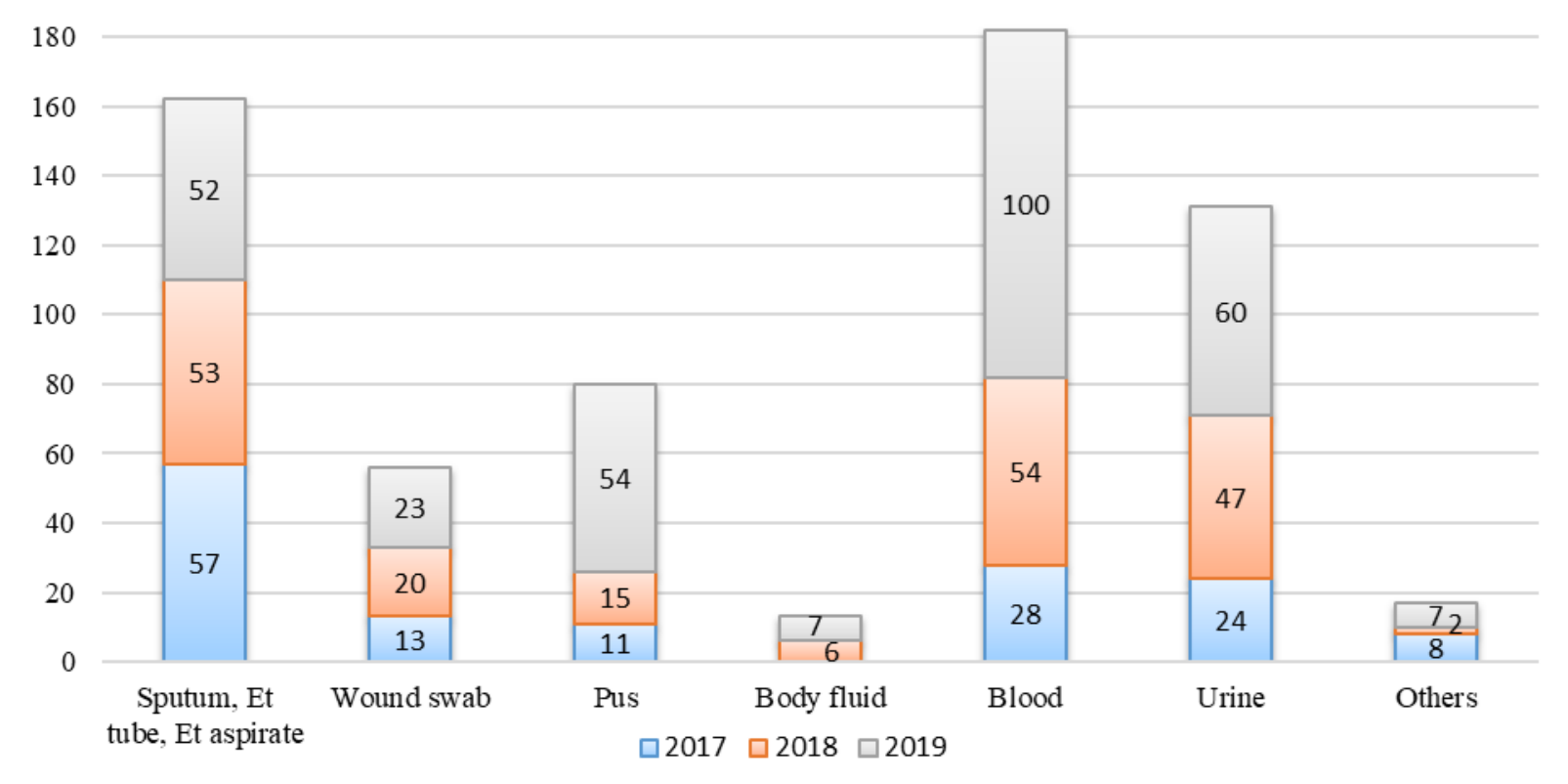

Figure: 1 Isolation of Pseudomonas spp from different samples 
Maximum number of isolates were from blood, followed by respiratory samples and urine samples (figure 1) though these differences were not significant statistically.

The antibiotic susceptibility profile revealed gradually decreasing number of resistant isolates over the period starting from 2017 to 2019 except for imipenem to which the organisms exhibited an increasing resistance trend. The percentage resistance of isolates against imipenem showed slow but gradual increase $(6.20 \%, 8.05 \%$ and $8.40 \%$ respectively in the years 2017,2018 and 2019) over the years. The number of MDR isolates were 32(22.69\%), 31(15.73\%) and 37(12.21\%) in the years 2017, 2018 and 2019 respectively (p-value $<0.05$ ).

Table 2 : Antibiotic resistance pattern in 2017, 2018 and 2019

\begin{tabular}{l|l|l|l|l|}
\cline { 2 - 5 } & $\mathbf{2 0 1 7}(\mathbf{R} \%)$ & $\mathbf{2 0 1 8}(\mathbf{R} \%)$ & $\mathbf{2 0 1 9}(\mathbf{R} \%)$ & p-value \\
\hline $\begin{array}{l}\text { Ciprofloxacin } \\
\text { Cefoperazone } \\
\text { sulbactam }\end{array}$ & 25.20 & 11.63 & 15.85 & $>0.05$ \\
\hline Ceftazidime & 43.18 & 24.28 & 13.26 & $<0.05$ \\
\hline $\begin{array}{l}\text { Piperacillin- } \\
\text { tazobactam }\end{array}$ & 58.33 & 62.07 & 39.39 & $>0.05$ \\
\hline Gentamicin & 26.47 & 35.63 & 11.88 & $<0.05$ \\
\hline Tobramycin & 38.46 & 16.67 & 13.43 & $>0.05$ \\
\hline Amikacin & 16.24 & 13.14 & 11.83 & $<0.05$ \\
\hline Imipenem & 6.20 & 8.05 & 6.25 & $>0.05$ \\
\hline Colistin & 3.30 & 0.67 & 8.40 & $>0.05$ \\
\hline Polymixin B & 0.00 & 0.00 & 0.60 & $>0.05$ \\
\hline $\begin{array}{l}\text { Multidrug re- } \\
\text { sistant isolates } \\
\text { (MDR) }\end{array}$ & $32(22.69 \%)$ & $31(15.73 \%)$ & $37(12.21 \%)$ & $<0.05$ \\
\hline
\end{tabular}

\section{DISCUSSION}

Pseudomonas spp is an important agent of hospital acquired as well as community acquired infections. It causes bacteremia, wound infection, respiratory tract infection, urinary tract infection etc. The outcome of infections is also associated with increased morbidity and mortality.

In this study, increase in number of Pseudomonas spp infection in consecutive years was observed. In all 3 years, total number of males affected were 19,754 and females were 21,228. Out of these, number of males whose specimens yielded Pseudomonas on culture were 385, a figure that was higher than the comparable figure among the females $(n=256)$. Though this overall difference was not a significant one ( $p$-value $>0.05$ ), yet, it was an interesting observation of male predominance of getting disease. Yadav et al have documented similar observation of male patients getting more Pseudomonas spp infections when compared to females ${ }^{5}$. The reason could be multifactorial but the important factor could be males were found to be less compliant to hand hygiene practice in terms of timing, use of soap, and hand washing behavior as compared to females ${ }^{11,12}$. Moreover, other factors like cultural, hormonal, immune status could also be related to male predominance of not only getting the disease, but also landing up with poor clinical outcome as well ${ }^{13}$.

In the present study, Pseudomonas spp was isolated starting from neonates to individuals as old as or $>80$ years of age. It was observed that majority of Pseudomonas spp $(304 ; 47.42 \%)$ were obtained from patients of the adult age group, whereas there were comparatively lesser numbers $(194 ; 30.26 \%)$ from those belonging to the old age group. In spite of the fact that, the older age group category needs to be managed with optimum care as these individuals are often vulnerable to other co-morbid conditions and are prone to infections as a result of seasonal climatic variations $^{14}$. 
In Pokhara, rainy season extends from May to October $^{15}$. As, Pseudomonas spp is a saprophytic organism, its dissemination is expected to be more in rainy season causing high number of infectious episodes ${ }^{16}$. In our study, 24,298 cultures were performed in rainy season and 17,355 during the non-rainy season. It was of note that Pseudomonas spp isolation rate was higher in the rainy months i.e. 371(57.8\%) when compared to the dry parts of the year $(270 ; 42.12 \%)$. Though this difference was not statistically significant, ( $p$-value $>0.05$ ), yet it was a notable difference which might have implications for the population in the community, especially for those in the old and much older age group.

Antibiotic resistance is the most feared aspect of bacterial infections. WHO warned it as a serious global situation. It was interesting and good to note that the dominance of antibiotic resistant Pseudomonas spp was in a decreasing trend (Table 2). Even the Multidrug resistant pseudomonas was on a decreasing trend ( $p$ value $<0.05$ ), as shown by the present study. This declining trend in antibiotic resistance exhibited a statistically significant difference (pvalue $<0.05$ ), especially with respect to antibiotics, such as cefoparazone sulbactam, piperacillin-tazobactam, tobramycin (table 2). This observation of ours was amply supported by the findings shown elsewhere ${ }^{17}$.

However, resistance rates seen in our study against ceftazidime, an antipseudomonal cephalosporin, was quite high varying between $58.33 \%$ in 2017 to $62.07 \%$ in 2018 . This seems to be alarming. According to recent studies from Nepal as well as from other countries, cephalosporins were one of the most widely used antibiotics for many deep seated infections and other infections, as clinician prescribed and as self-used antibiotic ${ }^{18,19}$.

Thus, our results could possibly implicate the rational use of cephalosporins in order to avoid rapid development of resistance to this drug by notorious bugs like Pseudomonas. Quinolone use and development of multidrug resistance were previously found to be interrelated ${ }^{20}$. In this context, it is important to note that ciprofloxacin is increasingly being used as a prescribed as well as a self-medicated antibiotic $^{19,21}$. Beside these, resistance to $\beta$-lactam antibiotics is governed by common mechanism ie $\beta$-lactamase production which can be trans- ferred within same or different bacterial species which might have contributed in higher resistance to $\beta$-lactam antibiotics.

Progressive increase in the resistance to imipenem, one of the last resort antibiotics, was noticed. However, it was seen in $7.55 \%$ of the isolates only, suggesting it could still be still a reliable antibiotic for treatment of multidrug resistant Pseudomonas spp infection. In contrast to our observation, authors within Nepal have mixed observation e.g. Gyawali et al in 2020 had observed $32.1 \%$ isolates resistant to Meropenem while Chander et al in 2018 had observed all the Pseudomonas isolates were sensitive to Imipenem ${ }^{22,23}$. However, our isolates were mostly sensitive to WHO reserved antibiotics colistin and polymyxin B. The overall resistance to colistin was only $1.52 \%$ and the resistance rate was decreasing over the years. All the Pseudomonas spp isolated were sensitive to polymyxin B. It is a heartening observation. Colistin (polymyxin E) and polymyxin $\mathrm{B}$ belong to same group polymyxin with similar structure, mechanism of action and even cross resistance. Different authors from Nepal and from other countries had reported colistin resistance varying from as low as $2 \%$ to as high as $21.3 \%{ }^{24,25}$. Despite their systemic toxicity colistin and polymyxin B are regarded as the ultimate therapeutic option for critically ill patients due to gram negative bacterial infection $^{26}$.

As per the present data, less than $20 \%$ Pseudomonas spp were found to be resistant to ciprofloxacin and aminoglycosides like gentamicin, tobramycin, amikacin. Amikacin was found best choice with resistance rate $11.87 \%$. The resistance rate of these antibiotics has decreased in 2019 when compared with 2017 suggesting these WHO watch group of antibiotics are still better option for management of infection by Pseudomonas spp.

Our antibiotic susceptibility pattern of Pseudomonas isolates shows increase in susceptibility in recent years to plenty of antibiotics tested. Such a trend is favorable. In our study, factors that might have played the role in the increase in susceptibility pattern to certain antibiotics were not looked into. Even then the important observations we made certainly pave the way for further research. 


\section{CONCLUSION}

The rate of infection of Pseudomonas spp is significantly increasing. Emergence of antibiotic resistance is multifactorial, similarly decrease in antibiotic resistance trait could be multifactorial. The resistance to cephalosporin was alarming. There was a significant decreasing trend in the resistance rates of the isolates to cefoparazone sulbactam, piperacillintazobactam, and tobramycin. In this era of antibiotic resistance, this is a favorable trend. The possible factors playing role have to be further studied, identified and promoted. Judicious use of these antibiotics should be continued.

\section{CONFLICT OF INTEREST}

None declared

\section{REFERENCES}

1. Fazeli H, Akbari R, Moghim S, Narimani T, Arabestani MR, Ghoddousi AR. Pseudomonas aeruginosa infections in patients, hospital means, and personnel's specimens. J Res Med Sci. 2012 Apr;17(4):332-7.

2. Rocha AJ, Barsottini MRO, Rocha RR, Laurindo MV, Moraes FLL, Rocha SL. Pseudomonas aeruginosa: Virulence Factors and Antibiotic Resistance Genes. Braz. arch. biol. Technol. 2019; 62:1-15. e19180503. Doi: https://doi.org/10.1590/1678-4324 $-2019180503$.

3. Pang Z, Raudonis R, Glick BR, Lin TJ, Cheng Z. Antibiotic resistance in Pseudomonas aeruginosa: mechanisms and alternative therapeutic strategies. Biotech adv. 2019 Jan-Feb; 37(1):177-92. Doi: https://doi.org/10.1016/j.biotechadv.2018.11.013

4. Baral S, Pokharel A, Subramanya SH, Nayak N. Clinico-epidemiological profile of Acinetobacter and Pseudomonas infections, and their antibiotic resistant pattern in a tertiary care center, Western Nepal. Nepal J Epidemiol. 2019;9(4):804-11. Doi: 10.3126/nje.v9i4.26962

5. Yadav SK, Sharma S, Mishra SK, Sherchand JB. Tale of $\beta$-Lactamases and Multidrug Resistance in Pseudomonas aeruginosa isolated from Inpatients in a University Hospital. JIOM Nepal. 2021 Apr;43 (1):19-24. DOI: https://doi.org/10.3126/ jiom.v43i1.37467

6. Temkin E, Adler A, Lerner A, Carmeli Y. Carbapenem-resistant Enterobacteriaceae: biology, epidemiology, and management. Ann N Y Acad Sci 2014;1323:22-42. Doi: 10.1111/nyas.12537. PMID: 25195939.

7. Collee, J. G., Mackie TJ, McCartney JE. Mackie \& McCartney Practical Medical Microbiology. 14th ed. New York: Churchill Livingstone. 1996.

8. CLSI. Performance Standards for Antimicrobial Susceptibility Testing. 27th ed. CLSI supplement M100. Wayne, PA: Clinical and Laboratory Standards Institute; 2017.

9. Antimicrobial Susceptibility Systems. Hi-Media Laboratories, Mumbai. Available from: https://
www.himedialabs.com/HML/images/literature/ pdf/100000027/68.pdf

10. Magiorakos A, Srinivasan A, Carey RB, Carmeli Y, Falagas ME, Giske CG et al. Multidrugresistant, extensively drug-resistant and pandrug-resistant bacteria: an international expert proposal for interim standard definitions for acquired resistance. ClinMicrobiol Infect 2012;18:268-81. DOI: https:// doi.org/10.1111/j.1469-0691.2011.03570.x

11. Garbutt C, Simmons G, Patrick D, Miller T. The public hand hygiene practices of New Zealanders: a national survey. N Z Med J 2007 Nov 9;120:U2810. PMID: 18264189

12. Mocker M, Liang MC, Champlin S. "Think the sink:" preliminary evaluation of a handwashing promotion campaign. Am $\mathrm{J}$ Infect Control 2013;41:275-7. Doi: 10.1016/j.ajic.2012.03.023.

13. Lunzen JV, Altfeld M. Sex Differences in Infectious Diseases-Common but Neglected, J Infect Dis. 2014 July 1;209(3):S79-80. Doi: https:// doi.org/10.1093/infdis/jiu159

14. Carnes BA, Staats D, Willcox BJ. Impact of climate change on elder health. $J$ Gerontol $A$ Biol Sci Med Sci. 2014;69(9):1087-91. Doi:10.1093/gerona/ glt159

15. Weather and climate. Climate in Pokhara, Nepal [Internet]. Amsterdam, Netherlands. Weather and climate Information. 18 Oct 2020. Available from: https://weather-and-climate.com/average-monthlyRainfall-Temperature-sunshine,Pokhara,Nepal

16. Gerba CP. Environmentally Transmitted Pathogens. Environmental Microbiology. 2015:509-50. Doi: 10.1016/B978-0-12-394626-3.00022-3.

17. Joseph MN, Devi S, Shashikala P, Kanungo R. Changing trend in the antibiotic resistance pattern of Pseudomonas aeruginosa isolated from wound swabs of out-patients and in-patients of a tertiary care hospital. J. clin. diagn. res. 2013 Oct 5;7(10): 2170-2. Doi: $10.7860 / J C D R / 2013 / 6113.3461$

18. WHO report on surveillance of antibiotic consumption: 2016-2018 early Implementation. Geneva: World Health Organization; 2018.

19. Nepal G, Bhatta S. Self-medication with Antibiotics in WHO Southeast Asian Region: A Systematic Review. Cureus. 2018 april 5;10(4): 2-17. Doi:10.7759/cureus. 2428

20. Conley ZC, Bodine TJ, Chou A, Zechiedrich L. Wicked: The untold story of ciprofloxacin. PLoS Pathog. 2018 Mar 1;14(3):1-13. Doi: 10.1371/ journal.ppat.1006805.

21. Cook PP, Gooch M, Rizzo S. Reduction in fluoroquinolone use following introduction of ertapenem into a hospital formulary is associated with improvement in susceptibility of Pseudomonas aeruginosa to group 2 carbapenems: a 10-year study. Antimicrob Agents Chemother. 2011 Dec;55 (12):5597-601. Doi: 10.1128/AAC.00742-11.

22. Gyawali R, Khadka RB, Shrestha B, Manandhar S. Antimicrobial susceptibility patterns of pseudomonas species isolated from various clinical samples at a tertiary care hospital. J Ins of Sci and Tech. 2020;25(2): 49-54. Doi: https://doi.org/10.3126/ jist.v25i2.33734 
23. Chander A, Shahid RM. Antimicrobial susceptibility patterns of Pseudomonas aeruginosa clinical isolates at a tertiary care hospital in Kathmandu, Nepal. Asian J Pharm Clin Res. 2013;6(3):235-8

24. Paudel A., Devkota SP, Shrestha A, Shah AK. Prevalence of Colistin-resistant Gram-negative Isolates Carrying the mcr-1 Gene among Patients Visiting a Tertiary Care Center. J Nep Med Assoc. 2020;58 (232):983-7. Doi: https://doi.org/10.31729/ jnma.5246.

25. Abd El-Baky RM, Masoud SM, Mohamed DS, Waly NGFM, Shafik EA, Mohareb DA, Elkady A, Elbadr MM, Hetta HF. Prevalence and Some Possible Mechanisms of Colistin Resistance Among Multidrug-Resistant and Extensively Drug-Resistant Pseudomonas aeruginosa. Infect Drug Resist. 2020;13:323-32. Doi: https://doi.org/10.2147/ IDR.S238811

26. Nation RL, Velkov T, Li J. Colistin and polymyxin B: peas in a pod, or chalk and cheese? Clin Infect Dis. 2014;59(1):88-94. Doi:10.1093/cid/ciu213 